\title{
Dedicated Lead for Occipital Nerve Stimulation for Treatment of Refractory Head Pain: Technical Note
}

\author{
Iris Smet, MD, Ali Jerjir, MD, Marieke Devos, $M_{S C}^{*}$ (D) and Jean-Pierre Van Buyten, MD, PhD

Multidisciplinary Pain Centre AZ Nikolaas, Belgium

\begin{abstract}
Objective: Occipital nerve stimulation (ONS) has been used for many years for the treatment of refractory headache and head pain. Its effect has been documented in case series and cohort studies. The positive clinical benefits, however, have been accompanied by high complication rates, predominantly due to lead migration and breakage. We report our institutional experience with performing occipital nerve stimulation using a dedicated, custom made, ONS lead.
\end{abstract}

Methods: We retrospectively reviewed the medical records of patients treated for their head pain with this dedicated ONS lead. Demographic information including age, gender, preoperative diagnosis, progression to permanent implant after a successful trial and technical complications, such as lead breakage and skin erosion, were collected.

In addition, therapy outcomes such as pain relief, degree of satisfaction with the treatment and analgesic use were collected.

\begin{abstract}
Results: A total of 71 patients with refractory headache disorders were implanted with the custom made ONS leads. The majority of patients $(65 / 71)$ had a successful percutaneous trial and proceeded to implant of the neurostimulator. The procedure was well tolerated by the patients and no lead migrations and skin erosions occurred during the 28 months (range 0-58) follow-up. Two patients had an infection and in three patients the system was explanted due to lack of longterm benefit.
\end{abstract}

Conclusions: The use of the dedicated ONS lead provides a safe and stable clinical solution for occipital nerve stimulation in patients with refractory head pain.

\section{Keywords}

Chronic head pain, Headache disorders, Occipital nerve stimulation, Tined lead, Adverse events

\section{Introduction}

Headache and head pain have a debilitating effect on patients often resulting in chronic pain and severe functional impairment. Despite significant developments in understanding and treating primary headaches and other head pain related indications, there remains a group of patients with difficult to treat head pain, labelled generically as medically refractory chronic headache $[1,2]$. In general terms, these patients have frequent, daily and near-daily headache unresponsive to medical therapy. The use of neurostimulation such as occipital nerve stimulation (ONS) has been described for the treatment of refractory headache or head pain [3-8]. Neurostimulation therapies use mild electrical stimulation methods and it may be applied to virtually any neural structure, including the spinal cord and peripheral nerves.

In a landmark paper, Weiner and Reed described the first results with ONS in 12 patients with occipital neuralgia, migraine and hemicrania continua [9]. ONS therapy consists of applying mild electrical stimulation to the nerves via leads implanted under the skin, near the occipital nerves at the base of the head. The leads are connected to an implantable programmable neurostimulator that includes a battery to generate the electrical stimulation pulses.

Although ONS has shown good results for control of chronic head pain and headache disorders, the relatively high adverse event rate, and lead dislocation and skin perforation in particular, forms a considerable limitation for broader acceptance of this therapy [10]. The high complication rates were often due to the use of unadapted lead hardware, specifically developed for use in the epidural space for spinal cord stim-

*Corresponding author: Marieke Devos, MSc, Pain Center AZ Nikolaas, Moerlandstraat 1, 9100 Sint-Niklaas, Belgium, Tel: $+32-3-760-23-90$

Accepted: October 20, 2020

Published online: October 22, 2020

Citation: Smet I, Jerjir A, Devos M, et al. (2020) Dedicated Lead for Occipital Nerve Stimulation for Treatment of Refractory Head Pain: Technical Note. J Neurosurg Res Rev 3(1):35-39 


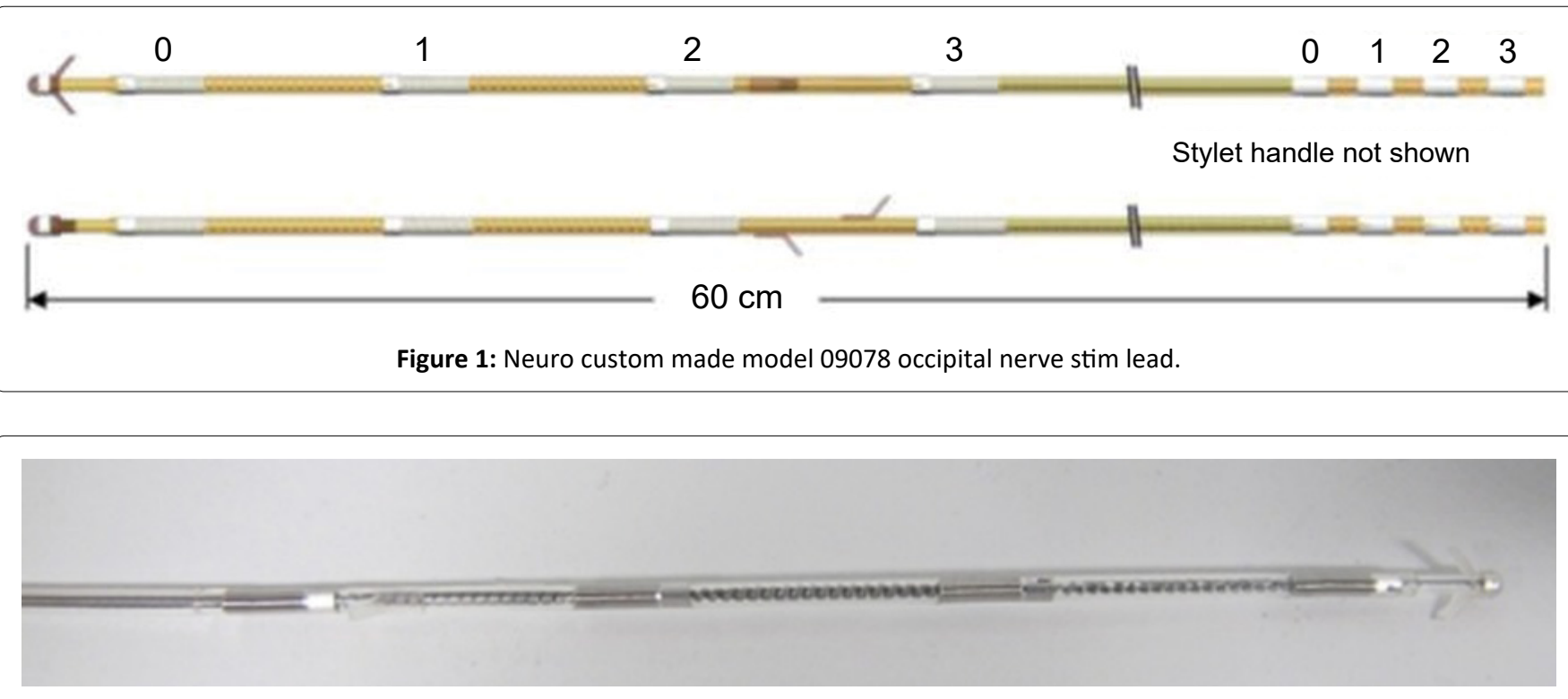

Figure 2: Neuro Custom-Made Model 09078 Occipital Nerve Stim Lead (Medtronic Bakken Research Center, Maastricht, Netherlands).

ulation (SCS). Similar technical complications were observed in our center in patients receiving ONS. This led us to look for alternative lead options and additional devices that would suit placement in the curved and motile area in the occipital region.

In this article we describe our experiences with a dedicated, "custom made" lead and introducer bended needle in patients with refractory head pain receiving ONS therapy.

\section{Operative Technique}

In order to improve fixation of the lead, while preventing skin irritation by movement of the lead in this motile area of the head/neck region, a dedicated, custom made lead for occipital nerve stimulation (Percutaneous Subcutaneous Quadripolar Occipital Nerve Stim Lead, Model 09078) was developed in cooperation with Medtronic (Bakken Research Center, Maastricht, Netherlands). Compared to the leads used for epidural SCS, the custom ONS lead contains 4 flexible electrodes in order to improve overall lead flexibility and also silicon tines to prevent dislocation (Figure 1 and Figure 2). The lead is placed with a 14 gauge curved introducer needle to follow the countoures of the occipital area.

Initially, when we started with ONS-therapy in our center, we used the implant technique as described by Weiner and Reed [9]. A subcutaneous lead was inserted towards the midline via a lateral incision close to the mastoid process. The procedure was performed under propofol sedation with a wake up during the procedure in order to check the correct position of the lead by electrical stimulation and the generated field of paresthesia. With growing experience in our center, the technique was adapted and the ONS implant procedure is since 2010 performed under general anesthesia with the patient in the prone position and the head in a horseshoe headrest.

For the surgery a first incision is made just below the occiput, where there is more subcutaneous fat tissue that allows for a pocket for a small loop of the lead. The curved needle is then inserted and pushed from the occiput towards the mastoid process in the subcutaneous tissue, to span the greater and lesser occipital nerves. The needle-stylet is then removed, and the lead introduced into the needle. Thereafter, the correct position of the lead is controlled with fluoroscopy and the introducer needle is retracted, leaving the tined lead in place. Once the lead is positioned correctly the lead-stylet is removed and a strain-relief loop created and placed in the pocket without anchoring the lead.

The tunneling tool is then used to subcutaneously pass the lead along the midline just next to the spine, downwards through the neck towards the extension connection site on the back of the patient. Another incision may be needed along the tunneled path, in between the lead and the extension site. At least 2 strain-relief loops are made along the lead trajectory by coiling the lead body into a circular loop greater than $2 \mathrm{~cm}$, each inside a $2-3 \mathrm{~cm}$ diameter subcutaneous pocket, to avoid inadvertent dislodgement by possible traction.

For the trial period, a temporary extension cable is tunneled laterally over the thoracic wall, connected to the lead(s) and externalized and connected to an external neurostimulator. Stimulation parameters, including frequency, pulse width and voltage, are adjusted so that patients experience mild paresthesia in the painful area. All patients receive trial stimulation for a period of at least 4 weeks after which the clinical effect is assessed. The duration of the trial allowes sufficient time for a clinical assessment of the therapy. Clinical success at end of trial period is defined as either $\geq 50 \%$ pain relief, a decrease in pain medication, a decrease in headache attacks or a decrease in headache days/month.

After a successful trial, the temporary extension cable is removed and a pocket created in the gluteal area for positioning of an implantable pulse generator (IPG). A new extension cable for connecting the ONS lead and the IPG, is tunneled towards the connector and secured to the underlying tissue. 
Citation: Smet I, Jerjir A, Devos M, et al. (2020) Dedicated Lead for Occipital Nerve Stimulation for Treatment of Refractory Head Pain: Technical Note. J Neurosurg Res Rev 3(1):35-39

Table 1: Patient characteristics and head pain diagnosis.

\begin{tabular}{|l|l|l|}
\hline & Total population $\mathbf{( n = 7 1 )}$ & Patients with permanent implant $\mathbf{( n = 6 5 )}$ \\
\hline Gender & 23 male/48 female & 20 male/45 female \\
\hline Age at implant of ONS lead (year) & Mean: 49 (23-71 y) & Mean: $49(23-71$ y) \\
\hline FNSS/Cervicogenic Headache & 35 & 32 \\
\hline Cluster headache & 14 & 13 \\
\hline Cervicogenic headache (ICHD-II 13.12) & 16 & 14 \\
\hline Atypical hemifacialgia/headache & 1 & 1 \\
\hline SUNCT & 1 & 1 \\
\hline Chronic migraine & 2 & 2 \\
\hline Hemicrania continua & 1 & 1 \\
\hline Post-surgery neuropathy & 1 & 1 \\
\hline
\end{tabular}

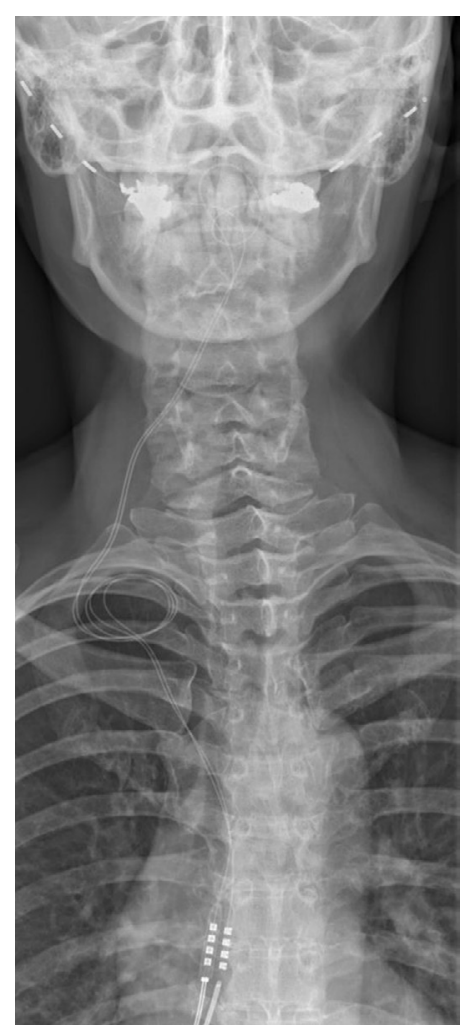

Figure 3: RX bilateral implant with custom-made ONS lead.

Figure 3 shows an X-ray of a bilateral implant with the custom made lead (Percutaneous Subcutaneous Quadripolar Occipital Nerve Stim Lead, Model 09078).

\section{Study Population}

We received approval from our local Ethics Committee for this study (EC 16025). Patients signed an informed consent form before completing the questionnaires related to the ONS therapy.

Data were retrospectively reviewed from electronic medical records from patients who had undergone ONS implants with this dedicated lead between 2013-2017 at the Multidisciplinary Pain Centre AZ Nikolaas.

The primary outcome was the need for explantation be- cause of complications such as lead dislocation, lead breakage and skin erosion. Other therapy-related side effects and complications were also recorded.

Furthermore, patients were asked to complete a questionnaire related to the ONS therapy they received. This questionnaire collected information on patients' head pain severity (10-point Visual Analogue Scale), degree of satisfaction with the treatment (5-point Likert scale) and analgesic use.

\section{Results}

A total of 71 patients with refractory head pain or headache disorders and who received ONS-therapy were included in this study. The clinical diagnosis was based on the symptoms and neurological assessment, including medical imaging to exclude tumors or underlying vascular diseases.

All patients had documented failure (lack of pain relief or intolerable side effects) of treatment with pharmacological agents (e.g. tricyclic antidepressants, neuroleptics, anticonvulsants, opioids, beta blockers, $\mathrm{Ca}^{+}$channel blockers). Most patients also had received one or more peripheral nerve blocks or radiofrequency procedures without benefits.

The majority of patients $(65 / 71 ; 91 \%)$ had a successful ONS trial according to the above criteria and proceeded to permanent implant of a neurostimulator. The six patients who did not benefit from trial ONS, had their ONS lead(s) explanted and exited the study.

Twelve patients had earlier received ONS-therapy with quadripolar SCS leads and had received the custom ONS lead when their original lead(s) failed due to technical complications (lead fracture or dislocation).

The patient characteristics and head pain diagnosis are described in Table 1. The majority of patients had failed neck surgery syndrome $(49 \%)$, cervicogenic headache $(23 \%)$ or cluster headache $(20 \%)$.

Patients suffering from bilateral head pain received bilateral lead implantation. The distribution of the implanted leads is illustrated in Table 2.

\section{Safety}

Overall follow-up time with the custom ONS-lead was on 
Table 2: Distribution of the implanted leads.

\begin{tabular}{|l|l|l|}
\hline & $\begin{array}{l}\text { Total } \\
\text { population } \\
(\mathbf{n = 7 1 )}\end{array}$ & $\begin{array}{l}\text { Patients with } \\
\text { permanent implant } \\
(\mathbf{n}=\mathbf{6 5})\end{array}$ \\
\hline Bilateral tined ONS leads & 40 & 37 \\
\hline Unilateral tined ONS lead & 31 & 28 \\
\hline
\end{tabular}

*One patient has a custom ONS-lead placed supra-orbitally.

Table 3: Patient outcome.

\begin{tabular}{|l|l|}
\hline Outcome & Number of patients \\
\hline Very satisfied & 28 \\
\hline Satisfied & 17 \\
\hline Moderate & 8 \\
\hline Failed & 4 \\
\hline Explanted & 4 \\
\hline Lost to follow-up & 2 \\
\hline Redirected & 2 \\
\hline
\end{tabular}

average 28 months (range $0-58$ ).

No lead migrations or skin erosions occurred with the custom ONS lead and the procedure was well tolerated by the patients. The following side effects and complications occurred in the population of patients with IPG implant: Two patients had an infection. One patient had an infection at the location of the IPG. Both the ONS leads and IPG were explanted and a new system was implanted after resolution of the infection. This patient has now received ONS for several years during which no further complications were observed. The second patient had an infection at the connector-site during the externalized trial phase after which both the ONS lead and extension cable were removed. After 3 months a new custom ONS lead was implanted and directly connected to an IPG without a new trial period.

In three patients the ONS system was explanted during the follow-up phase. The first patient didn't use the stimulator since she stopped working and asked for an explant. A second patient had an explant in another center due to loss of efficacy and one patient had the system explanted as a result of high stimulation amplitudes, due to fibrosis around the lead.

\section{Therapy outcomes}

The mean head pain score at baseline was $8.4(n=59)$ and $3.4(n=56)$ at last follow-up.

Sixty-six percent of patients $(n=43)$ had reduced their analgesic headache medication at last follow-up and 69\% (45/65) of patients who received a permanent IPG were satisfied or very satisfied with the treatment outcome. Table 3 provides an overview of patient outcome.

The majority of patients, $47 / 65$ patients (72\%), would choose to receive this treatment again.

\section{Discussion}

Chronic headache refractory to conservative treatment is invalidating and several studies have shown that neurostimulation of the occipital nerve helps to reduce pain and improve quality of life [11-14].

The most frequently reported complication, however, is lead migration that, depending on the research is reported to occur between 0 to $100 \%$ of the implantation [15]. Lead migration has been identified with the inadapted design of the lead commonly used for spinal cord stimulation. To prevent this we used a dedicated ONS lead with flexible electrodes to facilitate for movements of the lead in this motile area in the head/neck region, and silocone tines to prevent dislocation without the need for anchoring of the lead. In our series of 65 patients who received permanent ONS-treatment, we observed no lead migration over a period up to 58 months after implant of the custom ONS leads.

In total, 5 patients had the device explanted. Two patients developed and infection that required explant of the device, which could have been caused by the prolonged percutaneous trial period with an external stimulator in Belgium. Three patients had the system explanted due to various reasons (no use anymore, loss of efficacy and fibrosis).

At last follow-up, ONS therapy had reduced head pain on average by almost $60 \%$, in combination with a reduction in headache medication. These findings are in line with other studies that reported clinical outcomes of ONS-therapy $[4,5,7,8]$. These findings are also in line with the high satisfaction rating of the patients with the therapy.

The custom made ONS lead model that is described in this technical note has now been replaced by another dedicated ONS lead, AnkerStim lead, with similar design and that received CE-mark in December 2016.

\section{Sources of Support}

This work has been supported financially by the Medtronic External Research Program.

\section{Authorship}

Dr. Smet assisted in conceiving the study and implanted several patients and reviewed the final manuscript.

Dr. Jerjir retrieved the data; drafted the manuscript.

Mrs. Devos performed the data analysis.

Dr. Van Buyten conceived the study protocol, guided the production of the custom made lead, implanted several patients, reviewed and edited the manuscript.

\section{Conflict of Interest}

Dr. Smet and Dr. Van Buyten serve as consultant for Medtronic and Abbott.

\section{References}

1. Paemeleire K, Van Buyten JP, Van Buynder M, et al. (2010) Phenotype of patients responsive to occipital nerve stimulation for refractory head pain. Cephalalgia 30: 662-673. 
2. Goadsby PJ, Schoenen J, Ferrari MD, et al. (2006) Towards a definition of intractable headache for use in clinical practice and trials. Cephalalgia 26: 1168-1170.

3. Schwedt TJ, Dodick DW, Trentman TL, et al. (2006) Occipital nerve stimulation for chronic cluster headache and hemicrania continua: Pain relief and persistence of autonomic features. Cephalalgia 26: 1025-1027.

4. Burns B, Watkins L, Goadsby PJ (2007) Treatment of medically intractable cluster headache by occipital nerve stimulation: Longterm follow-up of eight patients. Lancet 369: 1099-1106.

5. Magis D, Allena M, Bolla M, et al. (2007) Occipital nerve stimulation for drug-resistant chronic cluster headache: A prospective pilot study. Lancet Neurol 6: 314-321.

6. Magis D, Gerardy PY, Remacle JM, et al. (2011) Sustained effectiveness of occipital nerve stimulation in drug-resistant chronic cluster headache. Headache 51: 1191-1201.

7. Miller S, Watkins L, Matharu M (2017) Treatment of intractable chronic cluster headache by occipital nerve stimulation: A cohort of 51 patients. Eur J Neurol 24: 381-390.

8. Fontaine D, Blond S, Lucas C, et al. (2017) Occipital nerve stimulation improves the quality of life in medically-intractable chronic cluster headache: Results of an observational prospective study. Cephalalgia 37: 1173-1179.
9. Weiner R, Reed K (1999) Peripheral neurostimulation for control of intractable occipital neuralgia. Neuromodulation 1999: 217221.

10. Schwedt TJ, Green AL, Dodick DW (2015) Occipital nerve stimulation for migraine: Update from recent multicenter trials. Prog Neurol Surg 29: 117-126.

11. Weiner R (2006) Occipital neurostimulation (ONS) for treatment of intractable headache disorders. Pain Med 7: S137-S139.

12. Palmisani S, Al-Kaisy A, Arcioni R, et al. (2013) A six year retrospective review of occipital nerve stimulation practice--controversies and challenges of an emerging technique for treating refractory headache syndromes. J Headache Pain 14: 67.

13. Magown P, Garcia R, Beauprie I, et al. (2009) Occipital nerve stimulation for intractable occipital neuralgia: An open surgical technique. Clin Neurosurg 56: 119-124.

14. Schoenen J, Jensen RH, Lanteri-Minet M, et al. (2013) Stimulation of the sphenopalatine ganglion (SPG) for cluster headache treatment. Pathway $\mathrm{CH}-1$ : A randomized, sham-controlled study. Cephalalgia 33: 816-830.

15. Magis D, Schoenen J (2012) Advances and challenges in neurostimulation for headaches. Lancet Neurol 11: 708-719.

Copyright: (C) 2020 Smet I, et al. This is an open-access article distributed under the terms of the Creative Commons Attribution License, which permits unrestricted use, distribution, and reproduction in any medium, provided the original author and source are credited. 